\title{
Comparison Between Geometric-arithmetic Indices
}

\author{
Kinkar Chandra Das ${ }^{\mathrm{a}, *}$ and Nenad Trinajstić \\ ${ }^{a}$ Department of Mathematics, Sungkyunkwan University, Suwon 440-746, Republic of Korea \\ ${ }^{\mathrm{b}}$ The Rugjer Bošković Institute, Zagreb 10002, Croatia \\ RECEIVED OCTOBER 28, 2011; REVISED SEPTEMBER 12, 2012; ACCEPTED SEPTEMBER 28, 2012
}

\begin{abstract}
The concept of geometric-arithmetic indices (GA) was introduced in the chemical graph theory very recently. In this letter we compare the geometric-arithmetic indices for chemical trees, starlike trees and general trees. Moreover, we give a conjecture for general graphs. (doi: 10.5562/cca2005)
\end{abstract}

Keywords: Molecular Graph, Chemical Tree, Starlike Tree, Geometric-arithmetic Indices

\section{INTRODUCTION}

Let $G=(V, E)$ denote a simple graph with $n$ vertices and $m$ edges, $V(G)=\{1,2, \ldots, n\}$ and $m=|E(G)|{ }^{1}$ Also, let $d_{i}$ be the degree of the vertex $i$ for $i=1,2, \ldots, n$. The maximum vertex degree is denoted by $\Delta$ in $G$. Recently, a new class of topological descriptors, based on some properties of vertices of graph is presented. These indices are named as "geometric-arithmetic indices" $\left(G A_{\text {general }}\right)$ and their definition is as follows:

$$
G A_{\text {general }}=G A_{\text {general }}(G)=\sum_{i j \in E(G)} \frac{\sqrt{Q_{i} Q_{j}}}{\frac{1}{2}\left(Q_{i}+Q_{j}\right)}
$$

where $Q_{i}$ is some quantity that in a unique manner can be associated with the vertex $i$ of the graph $G$. The first member of this class was considered by Vukičević and Furtula ${ }^{2}$ by setting $Q_{i}$ to be the degree $d_{i}$ of the vertex $i$ of the graph $G$ :

$$
G A_{1}=G A_{1}(G)=\sum_{i j \in E(G)} \frac{2 \sqrt{d_{i} d_{j}}}{d_{i}+d_{j}} .
$$

For $i, j \in \mathrm{V}(\mathrm{G})$, let $d(i, j \mid G)$ be the distance between the vertices $i$ and $j$ in $G$. For $i j \in E(G)$,

$$
n_{i}=|\{x \in V(G): d(x, i \mid G)<d(x, j \mid G)\}|
$$

The second member of this class was considered by Fath-Tabar et al. ${ }^{3}$ by setting $Q_{i}$ to be the number $n_{i}$ of vertices of $G$ lying closer to the vertex $i$ than to the vertex $j$ for the edge $i j$ of the graph $G$ :

$$
G A_{2}=G A_{2}(G)=\sum_{i j \in E(G)} \frac{2 \sqrt{n_{i} n_{j}}}{n_{i}+n_{j}} .
$$

Let $x$ be a vertex and $i j$ be an edge of the graph $G$. The distance between $x$ and $i j$ is defined as

$$
d(x, i j \mid G)=\min \{d(x, i \mid G), d(x, j \mid G)\} .
$$

For $i j \in \mathrm{E}(\mathrm{G})$, let

$$
m_{i}=|\{f \in E(G): d(i, f \mid G)<d(j, f \mid G)\}| .
$$

It should be noted that $m_{i}$ is not a quantity that in a unique manner can be associated with the vertex $i$ of the graph $G$, but that it depends on the edge $i j$. Yet, this restriction is not relevant for the definition of $G A_{3}$. Note that in all cases $m_{i} \geq 0$ and

$$
m_{i}+m_{j} \leq m-1
$$

Then, incorporating $m_{i}$ as vertex quantity into Equation (1), the third geometric-arithmetic index is defined as, ${ }^{4}$

$$
G A_{3}=G A_{3}(G)=\sum_{i j \in E(G)} \frac{2 \sqrt{m_{i} m_{j}}}{m_{i}+m_{j}} .
$$

It has been demonstrated, on the example of octane isomers, that GA index is well-correlated with a variety of physico-chemical properties. ${ }^{2}$ Vukičević and Furtula $^{2}$ in order to study the predicive power of the GA index considered the following set of octane properties: boiling points, entropy, enthalpy of vaporization, stand-

\footnotetext{
* Author to whom correspondence should be addressed. (E-mail: kinkardas2003@googlemail.com)
} 
Table 1. Comparison of structure-property models based on the GA indices to models obtained by the connectivity index

\begin{tabular}{lcc}
\hline \multirow{2}{*}{ Property } & \multicolumn{2}{c}{ Correlation Coefficient } \\
\cline { 2 - 3 } & $G A$-index & Connectivity index \\
\hline Boiling point & 0.823 & 0.821 \\
Entropy & 0.912 & 0.906 \\
Enthalpy of vaporization & 0.941 & 0.936 \\
Standard enthalpy of & 0.966 & 0.958 \\
vaporization & 0.858 & 0.850 \\
Enthalpy of formation & 0.912 & 0.904 \\
Acentric factor &
\end{tabular}

ard enthalpy of vaporization, enthalpy of formation and acentric factor. The structure-property models based on the GA indices were comparable (and in some cases even better than) to models obtained by the connectivity index. ${ }^{5}$ This can be seen from data in Table 1, taken from the paper by Vukučević and Furtula. ${ }^{2}$

The mathematical properties and uses of geometricarithmetic indices are studied by several groups. ${ }^{2-4,6-13}$ A survey of mathematical properties of the GA indices and their uses in QSPR and QSAR is recently given by Das, Gutman and Furtula. ${ }^{14}$ The above results indicate the potential of the GA molecular descriptors in the structure-property-activity modeling. In order to fully explore their potential, it is necessary to study the mathematical and computational properties and the range of applicability of the GA indices. The preliminary results are encouraging. We compare the first geometricarithmetic index and the atom-bond connectivity index. $^{15}$ In this letter we compare the geometricarithmetic indices for chemical trees, starlike trees and general trees. Moreover, we give a conjecture for general graphs. Finally, we give conclusion.

\section{PRELIMINARIES}

A connected graph with maximum vertex degree at most 4 belongs to a family of molecular graphs depicting carbon compounds. ${ }^{16}$ Its graphical representation may resemble a structural formula of some (usually organic) molecule. That was a primary reason for employing graph theory in chemistry. Nowadays this area of mathematical chemistry is called chemical graph theory. ${ }^{16} \mathrm{~A}$ tree in which the maximum vertex degree does not exceed 4 is said to be a "chemical tree". A vertex of a graph is said to be pendent if its neighborhood contains exactly one vertex. An edge of a graph is said to be pendent if one of its vertices is a pendent vertex. Denote, as usual, by $K_{1, n-1}$ and $P_{n}$, the star and the path on $n$ vertices, respectively.

A tree is said to be starlike if exactly one of its vertices has degree greater than two. By $S\left(r_{1}, r_{1}, \ldots, r_{k}\right)$, we denote the starlike tree which has a vertex 1 of degree $k \geq 3$ and which has the property

$$
S\left(r_{1}, r_{2}, \ldots, r_{k}\right) \backslash\{1\}=P_{r_{1}} \cup P_{r_{2}} \cup \ldots \cup P_{r_{k}}
$$

This tree has $r_{1}+r_{2}+\ldots+r_{k}+1=n$ vertices and assumed that $r_{1} \geq r_{2} \geq \ldots \geq r_{k} \geq 1$. We say that the starlike tree $S\left(r_{1}, r_{1}, \ldots, r_{k}\right)$ has $k$ branches, the lengths of which are $r_{1}, r_{1}, \ldots, r_{k}$, respectively.

\section{COMPARISON BETWEEN $G A_{1}$ INDEX AND $G A_{2}$ INDEX}

In this section we compare between $G A_{1}$ and $G A_{2}$ index for chemical trees and starlike trees. First we prove the following result:

Lemma. Let $T$ be a chemical tree. Then the number of pendent vertices in $T$ are $2 a+b+2$, where $a$ is the number of four degree vertices and $b$ is the number of three degree vertices in $T$.

Proof: If $h_{i}$ is the number of vertices of degree $i$ in $T$, then we have

and

$$
h_{1}+h_{2}+h_{3}+h_{4}=n
$$

$$
h_{1}+2 h_{2}+3 h_{3}+4 h_{4}=2(n-1) \text {. }
$$

From the two relations above we get

$$
h_{1}-h_{3}-2 h_{4}=2 \text {, i.e. } h_{1}=2 a+b+2 \text {, }
$$

where $h_{3}=b$ and $h_{4}=a$. Hence the Lemma.

Now we compare between $G A_{1}$ index and $G A_{2}$ index for chemical tree $T$.

Theorem 1. Let $T$ be a chemical tree of order $n$. Then,

$$
G A_{1}(T) \geq G A_{2}(T)
$$

with equality if and only if $G$ is isomorphic to $K_{1, i}, i=1$, $2,3,4$.

Proof: If $T \cong K_{1, \mathrm{i}}, i=1,2,3,4$; one can see easily that $G A_{1}(T)=G A_{2}(T)$. If $T \cong P_{n}(n>3)$, then from the definition of $G A$ indices, we have $G A_{1}(T)>G A_{2}(T)$.

Otherwise, $3 \leq \Delta \leq 4, n \geq 5$, and $T \cong K_{1, i}, i=1,2$, 3,4 . Since $T$ is a chemical tree, we must have $1 \leq d_{i} \leq 4$ for all $i$. Thus we have the edges with possible degree pairs $(4,1),(4,2),(4,3),(4,4),(3,1),(3,2),(3,3),(2,1)$, $(2,2)$. In Table 2 , we calculate the values of $\frac{2 \sqrt{d_{i} d_{j}}}{d_{i}+d_{j}}$ for all above degree pairs. First we assume that $n \geq 10$. Let 
Table 2. Calculated values of $\frac{2 \sqrt{d_{i} d_{j}}}{d_{i}+d_{j}}$ for possible degree pairs

\begin{tabular}{cccccccccc}
\hline$\left(d_{i}, d_{j}\right)$ & $(4,1)$ & $(4,2)$ & $(4,3)$ & $(4,4)$ & $(3,1)$ & $(3,2)$ & $(3,3)$ & $(2,1)$ & $(2,2)$ \\
\hline$\frac{2 \sqrt{d_{i} d_{j}}}{d_{i}+d_{j}}$ & 0.8 & $\sqrt{\frac{8}{9}}$ & $\sqrt{\frac{48}{49}}$ & 1 & $\sqrt{\frac{3}{4}}$ & $\sqrt{\frac{24}{25}}$ & 1 & $\sqrt{\frac{8}{9}}$ & 1 \\
\hline
\end{tabular}

$a$ be the number of vertices of degree four and also let $b$ be the number of vertices of degree three in $T$. Then there are at most $4 a+3 b$ non-pendent edges $i j$ with not $d_{i}=d_{j}=2$. By Lemma, the number of pendent vertices are $2 a+b+2$ in $T$. From Table 2, we have

$$
\frac{2 \sqrt{d_{i} d_{j}}}{d_{i}+d_{j}} \geq 0.8
$$

for each pendent edge $i j \in E(T)$ and

$$
\frac{2 \sqrt{d_{i} d_{j}}}{d_{i}+d_{j}} \geq \sqrt{\frac{8}{9}}
$$

for each non-pendent edge $i j \in E(T)$.

Since $n \geq 10$, we have

$$
\frac{2 \sqrt{n_{i} n_{j}}}{n_{i}+n_{j}} \leq 0.6
$$

for each pendent edge $i j \in E(T)$ and

$$
\frac{2 \sqrt{n_{i} n_{j}}}{n_{i}+n_{j}} \leq 1
$$

for each non-pendent edg $i j \in E(T)$.

Using above results, we get

$$
\begin{aligned}
G A_{1}(T)-G A_{2}(T)= & \sum_{i j \in E(T), d_{j}=1}\left(\frac{2 \sqrt{d_{i} d_{j}}}{d_{i}+d_{j}}-\frac{2 \sqrt{n_{i} n_{j}}}{n_{i}+n_{j}}\right) \\
& +\sum_{i j \in E(T), d_{i}=d_{j}=2}\left(\frac{2 \sqrt{d_{i} d_{j}}}{d_{i}+d_{j}}-\frac{2 \sqrt{n_{i} n_{j}}}{n_{i}+n_{j}}\right) \\
& +\sum_{i j \in E(T), d_{j}>2}\left(\frac{2 \sqrt{d_{i} d_{j}}}{d_{i}+d_{j}}-\frac{2 \sqrt{n_{i} n_{j}}}{n_{i}+n_{j}}\right) \\
& \geq 0.2(2 a+b+2)-0.06(4 a+3 b)>0,
\end{aligned}
$$

as

$$
\frac{2 \sqrt{d_{i} d_{j}}}{d_{i}+d_{j}}=1 \geq \frac{2 \sqrt{n_{i} n_{j}}}{n_{i}+n_{j}} \text { for } d_{i}=d_{j}=2
$$

Next we have to show that $G A_{1}(T)>G A_{2}(T)$ for 5 $\leq n \leq 9$. If $n=5$, then $T \cong T_{1}$ (Figure 1) as $T \nRightarrow K_{1,4}, P_{5}$. For $T=T_{1}$, we have $G A_{1}(T)>G A_{2}(T)$. Otherwise, $6 \leq n$ $\leq 9$. Now we consider two cases (a) $\Delta=3$, (b) $\Delta=4$.

Case (a): $\Delta=3$. Using Table 2, we have

$$
\frac{2 \sqrt{d_{i} d_{j}}}{d_{i}+d_{j}}-\frac{2 \sqrt{n_{i} n_{j}}}{n_{i}+n_{j}}>0.866-0.746=0.12
$$

for each pendent edge $i j \in E(T)$, as $n \geq 6$ and

$$
\frac{2 \sqrt{d_{i} d_{j}}}{d_{i}+d_{j}}-\frac{2 \sqrt{n_{i} n_{j}}}{n_{i}+n_{j}}>0.97-1=-0.03
$$

for each non-pendent edge $i j \in E(T)$.

In this case there are at least three pendent edges in $T$. Using above results, we get $G A_{1}(T)>G A_{2}(T)$ as $n \leq 9$.

Case (b): $\Delta=4$. If $T \cong T_{2}$ (Figure 1 ), then one can see easily that $G A_{1}(T)>G A_{2}(T)$. Otherwise, $7 \leq n \leq 9$. In this case there are at least four pendent edges and at most four non-pendent edges in $T$ as $n \leq 9$. Using Table 2, we have

$$
\frac{2 \sqrt{d_{i} d_{j}}}{d_{i}+d_{j}}-\frac{2 \sqrt{n_{i} n_{j}}}{n_{i}+n_{j}}>0.8-0.7=0.1
$$

for each pendent edge $i j \in E(T)$, as $n \geq 7$ and

$$
\frac{2 \sqrt{d_{i} d_{j}}}{d_{i}+d_{j}}-\frac{2 \sqrt{n_{i} n_{j}}}{n_{i}+n_{j}}>0.94-1=-0.06
$$

for each non-pendent edge $i j \in E(T)$. Thus we get $G A_{1}(T)>G A_{2}(T)$. This completes the proof.

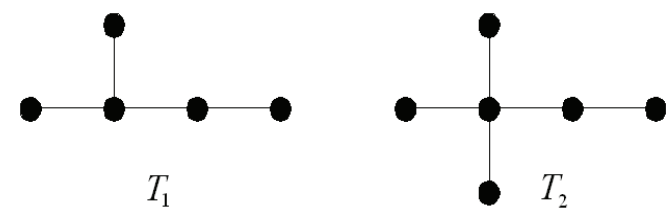

Figure 1. Two trees $\mathrm{T} 1$ and $\mathrm{T} 2$. 
Now we compare between $G A_{1}(T)$ index and $G A_{2}(T)$ index for starlike trees.

Theorem 2. Let $S\left(r_{1}, r_{1}, \ldots, r_{k}\right)$ be a starlike tree of order $n$. Then

$$
G A_{1}(S) \geq G A_{2}(S)
$$

with equality if and only if $S$ is isomorphic to star $K_{1, \mathrm{n}-1}$.

Proof: When $r_{1}=r_{2}=\ldots=r_{k}=1$, we have

$$
\frac{2 \sqrt{d_{i} d_{j}}}{d_{i}+d_{j}}=\frac{2 \sqrt{n-1}}{n}=\frac{2 \sqrt{n_{i} n_{j}}}{n_{i}+n_{j}}
$$

for any edge $i j \in E(S)$. Thus we have $G A_{1}\left(K_{1, \mathrm{n}-1}\right)=$ $G A_{2}\left(K_{1, \mathrm{n}-1}\right)$. Otherwise, $r_{l} \geq 2$.

First we assume that $r_{k} \geq 2$. Then $r_{1} \geq r_{2} \geq \ldots \geq r_{k} \geq$ 2 and $n \geq 7$. Thus we have the edges with possible degree pairs $(k, 1),(k, 2),(2,1),(2,2)$. For each edge $i j$ we have

$$
\frac{2 \sqrt{d_{i} d_{j}}}{d_{i}+d_{j}} \geq \frac{2 \sqrt{n_{i} n_{j}}}{n_{i}+n_{j}}
$$

except possibly edges ij with degree pair $(k, 2)$. Now we have

$$
\frac{\sqrt{2 k}}{k+2}>\frac{\sqrt{n-1}}{n}
$$

that is,

$$
2(n-1)-k+\frac{2}{n-1}>\frac{4}{k}
$$

which, evidently, is always obeyed. Also we have

$$
\frac{\sqrt{2}}{3}>\frac{\sqrt{2(n-2)}}{n},
$$

that is,

$$
n+\frac{4}{n-2}>7
$$

which, evidently, is always obeyed. Moreover, we have

$$
r_{i}\left(n-r_{i}\right) \leq \frac{n^{2}}{4} \text { for } 1 \leq i \leq k
$$

Now,

$$
G A_{1}(S)=\frac{2 \sqrt{2 k}}{k+2} k+\sum_{i=1}^{k}\left(r_{i}-2\right)+\frac{2 \sqrt{2}}{3} k
$$

and

$$
G A_{2}(S)=\sum_{i=1}^{k} \sum_{j=2}^{r_{i}} \frac{2 \sqrt{j(n-j)}}{n}+\frac{2 \sqrt{n-1}}{n} k
$$

$$
\begin{aligned}
& \leq \sum_{i=1}^{k}\left(r_{i}-2\right)+\frac{2 \sqrt{2(n-2)}}{n} k+\frac{2 \sqrt{n-1}}{n} k \\
& <\sum_{i=1}^{k}\left(r_{i}-2\right)+\frac{2 \sqrt{2}}{3} k+\frac{2 \sqrt{2 k}}{k+2} k \quad \text { by (4) and } r_{k} \geq 2 \\
& =G A_{1}(S) .
\end{aligned}
$$

Next we assume that some of $r_{i}$ are equal to 1 . Let $r_{1} \geq$ $r_{2} \geq \ldots \geq r_{q} \geq 2, r_{q+1}=r_{q+2}=\ldots=r_{k}=1$. Similarly, we can easily show that

$$
\sum_{i=1}^{q} \sum_{j=1}^{r_{i}} \frac{2 \sqrt{j(n-j)}}{n}<\frac{2 \sqrt{2 k}}{k+2} q+\sum_{i=1}^{q}\left(r_{i}-2\right)+\frac{2 \sqrt{2}}{3} q
$$

and

$$
\frac{\sqrt{k}}{k+1} \geq \frac{\sqrt{n-1}}{n} \text {. }
$$

From above results we have

$$
G A_{1}(S)>G A_{2}(S) \text {. }
$$

This completes the proof.

\section{COMPARISON BETWEEN $G_{2}$ INDEX AND $G A_{3}$ INDEX}

In this section we compare between $G A_{2}$ index and $G A_{3}$ index for any tree $T$.

Theorem 3. For any tree $T$,

$$
G A_{2}(T)>G A_{3}(T)
$$

Proof: For any tree $T$,

$$
n_{i}=m_{i}+1, n_{j}=m_{j}+1
$$

for any edge $i j \in E(T)$.

Without loss of generality, we can assume that $n_{i} \geq$ $n_{j}$ for any non-pendent edge $i j \in E(T)$, that is, we have

$$
\frac{n_{i}}{n_{j}}=\frac{m_{i}+1}{m_{j}+1} \leq \frac{m_{i}}{m_{j}}
$$

and

$$
\frac{n_{j}}{n_{i}} \geq \frac{m_{j}}{m_{i}} .
$$

From above results we have

$$
\left(\frac{n_{i}}{n_{j}}\right)^{\frac{1}{4}}-\left(\frac{n_{j}}{n_{i}}\right)^{\frac{1}{4}} \leq\left(\frac{m_{i}}{m_{j}}\right)^{\frac{1}{4}}-\left(\frac{m_{j}}{m_{i}}\right)^{\frac{1}{4}}
$$


Squaring both sides and simplifying, we get

that is,

$$
\sqrt{\frac{n_{i}}{n_{j}}}+\sqrt{\frac{n_{j}}{n_{i}}} \leq \sqrt{\frac{m_{i}}{m_{j}}}+\sqrt{\frac{m_{j}}{m_{i}}}
$$

$$
\frac{\sqrt{n_{i} n_{j}}}{n_{i}+n_{j}} \geq \frac{\sqrt{m_{i} m_{j}}}{m_{i}+m_{j}}
$$

for any non-pendent edge $i j \in E(T)$. But for any pendent edge $i j \in E(T)$,

$$
\frac{\sqrt{n_{i} n_{j}}}{n_{i}+n_{j}}>0=\frac{\sqrt{m_{i} m_{j}}}{m_{i}+m_{j}} .
$$

Thus we have $G A_{2}(T)>G A_{3}(T)$. This completes the proof.

Corollary. Let $T$ be a chemical tree or starlike tree of order $n$. Then

$$
G A_{1}(T)>G A_{3}(T)
$$

Proof: By Theorem 1, Theorem 2, and Theorem 3, we get the required result.

Finally, the following conjecture holds.

Conjecture. For any connected graph $G$,

$$
G A_{1}(T) \geq G A_{2}(T) \geq G A_{3}(T) .
$$

\section{CONCLUSION}

In this report we discuss the comparison between first and second geometric-arithmetic indices for chemical trees and starlike trees. Besides these, it has been shown that second geometric-arithmetic index is greater than to the third geometric-arithmetic index for any tree. Com- parison between these indices, in the case of general graphs, remains an open problem.

Acknowledgement. The first author is partially supported by the Faculty research Fund, Sungkyunkwan University, 2012 and Sungkyunkwan University BK21 Project, BK21 Math Modeling HRD Div. Sungkyunkwan University, Suwon, Republic of Korea. The second author is partially supported by the Ministry of Science, Education and Sports of Croatia.

\section{REFERENCES}

1. R. J. Wilson, Introduction to Graph Theory, Oliver \& Boyd, Edinburgh, 1974, 2nd printing.

2. D. Vukičević and B. Furtula, J. Math. Chem. 46 (2009) 1369-1376.

3. G. Fath-Tabar, B. Furtula, and I. Gutman, J. Math. Chem. 47 (2010) 477-486.

4. B. Zhou, I. Gutman, B. Furtula, and Z. Du, Chem. Phys. Lett. 482 (2009) 153-155.

5. (a) R. Todeschini and V. Consonni, Handbook of Molecular Descriptors, Wiley-VCH, Weinheim, 2000; (b) R. Todeschini and V. Consonni, Molecular Descriptors for Chemoinformatics, Wiley-VCH, Weinheim, 2009, Vol. I and II.

6. Y. Yuan, B. Zhou, and N. Trinajstić, J. Math. Chem. 47 (2010) 833-841.

7. K. C. Das, MATCH Commun. Math. Comput. Chem. 64 (3) (2010) 619-630.

8. K. C. Das, I. Gutman, and B. Furtula, Discrete Appl. Math. 159 (2011) 2030-2037.

9. K. C. Das, I. Gutman, and B. Furtula, Iranian J. Math. Chem. 1 (2) (2010) 17-28.

10. K. C. Das, I. Gutman, and B. Furtula, Iranian J. Math. Chem. 1 (2) (2010) 29-36.

11. M. Mogharrab and G. H. Fath-Tabar, MATCH Commun. Math. Comput. Chem. 65 (2011) 33-38.

12. I. Gutman and B. Furtula (Eds.), Novel Molecular Structure Descriptors - Theory and Applications I, University of Kragujevac, Kragujevac, Serbia, 2010 pp. 137-172.

13. B. Furtula amd I. Gutman, J. Chemometrics 25 (2011) 87-91.

14. K. C. Das, I. Gutman, and B. Furtula, MATCH Commun. Math. Comput. Chem. 65 (3) (2011) 595-644.

15. K. C. Das and N. Trinajstić, Chem. Phys. Lett. 497 (2010) 149-151.

16. N. Trinajstić, Chemical Graph Theory, CRC Press, Boca Raton, 1983; 2nd revised ed. 1992. 